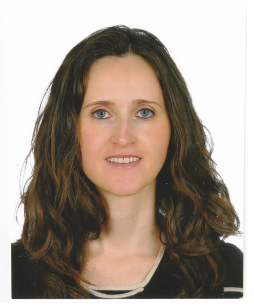

\title{
Catálogo de herramientas sobre la traducción y los dispositivos móviles
}

Inmaculada Serón-Ordóñez

Universidad Pablo de Olavide, de Sevilla

\section{RESUMEN}

El presente catálogo recoge herramientas relacionadas con las múltiples intersecciones entre la traducción y los dispositivos móviles; no está ceñido ni a procesos específicos ( $p$. ej., la traducción de aplicaciones o el uso de aplicaciones para traducir o interpretar) ni a terminales concretos. Se distinguen nueve tipos principales de herramientas, según su función: la consulta de material de referencia (diccionarios, corpus...), la gestión de la terminología, la gestión de tareas y proyectos, la toma de notas, la interacción con dispositivos (táctil, de voz,...), la edición de traducciones, la traducción automática y la posedición, la agregación de contenidos, y el desarrollo de aplicaciones móviles.

Palabras clave: dispositivos móviles, tabletas, teléfonos inteligentes, traducción, interpretación, localización, aplicaciones móviles, software

\section{ABSTRACT}

This catalogue includes tools related to the multiple intersections between translation and mobile devices; it is not limited to either specific processes (such as app translation or the use of apps to translate/interpret) or concrete terminals. Nine main types of tools are distinguished, according to their function: consultation of reference materials (dictionaries, corpora, etc.), terminology management, task and project management, note-taking, userdevice interaction (touch input, voice input, etc.), translation editing, machine translation and post-editing, content aggregation, and mobile app development.

Keywords: mobile devices, tablets, smartphones, translation, interpreting, localisation, mobile apps, software

\section{RESUM}

Aquest catàleg recull eines relacionades amb les múltiples interseccions entre la traducció i els dispositius mòbils; no es limita ni a processos específics (per exemple, la traducció d'aplicacions o l'ús d'aplicacions per a traduir o interpretar) ni a terminals concrets. Hi distingim nou tipus principals d'eines, segons la funció: la consulta de material de referència (diccionaris, corpus, etc.), la gestió de terminologia, la gestió de tasques i projectes, la presa de notes, la interacció amb dispositius (tàctil, de veu, etc.), l'edició de traduccions, la traducció automàtica i la postedició, l'agregació de continguts i el desenvolupament d'aplicacions mòbils

Paraules clau: dispositius mòbils, tauletes, telèfons intel-ligents, traducció, interpretació, localització, aplicacions mòbils, programari 


\section{Introducción}

La aparición de los dispositivos móviles, comenzando por las tabletas y los teléfonos inteligentes, está transformando el sector de la localización, al haber propiciado nuevos sistemas operativos y multitud de nuevas aplicaciones, los cuales, además, están influyendo en el software de escritorio y en los sitios web. Por otro lado, la tecnología móvil está modificando los modos de trabajo de los traductores e intérpretes.

El siguiente catálogo, concebido como un complemento al número de Tradumàtica dedicado a las intersecciones entre la traducción y los dispositivos móviles, recoge herramientas relacionadas con esta temática. Lejos de ser un catálogo exhaustivo, ofrece aplicaciones aportadas por profesionales y académicos a través de un formulario en línea que los editores del número difundimos mediante las redes sociales y a través de foros de traductores (como las listas de distribución de la Asociación Española de Traductores, Correctores e Intérpretes y de la División de Español de la American Translators Association). Los editores hemos revisado y completado la información aportada sobre cada aplicación. El formulario, disponible desde el 5 de noviembre hasta el 30 de diciembre de 2016, tenía como objetivo conocer de qué manera se están usando actualmente los dispositivos móviles en relación con la traducción y cuál puede ser su papel en el futuro. Por consiguiente, el catálogo no se ciñe a procesos específicos (como la traducción de aplicaciones o el uso de aplicaciones para traducir o interpretar) ni a terminales concretos.

Está estructurado en las siguientes categorías, según la función principal de las herramientas: consulta de material de referencia (diccionarios, corpus...); gestión de la terminología; gestión de tareas y proyectos; toma de notas; interacción con dispositivos (táctil, de voz,...); edición de traducciones; traducción automática y posedición; agregación de contenidos; desarrollo de aplicaciones móviles; otras. Incluye el nombre y los datos de contacto de la persona que aportó cada herramienta, siempre que se prestara conformidad para ello.

\section{Consulta de material de referencia}

\begin{tabular}{|l|l|}
\hline $\begin{array}{l}\text { Nombre de la } \\
\text { herramienta }\end{array}$ & Diccionarios del grupo Enciclopèdia Catalana \\
\hline Descripción breve & Diccionarios varios \\
\hline Enlace & http://grupenciclopedia.cat/diccionaris-de-lenciclopedia/ \\
\hline $\begin{array}{l}\text { Sistema(s) } \\
\text { operativo(s) }\end{array}$ & Varios \\
\hline Precio/Licencia & Varios \\
\hline Versión actual & \\
\hline Observaciones & \\
\hline Colaborador & \\
\hline
\end{tabular}

\begin{tabular}{|l|l|}
\hline $\begin{array}{l}\text { Nombre de la } \\
\text { herramienta }\end{array}$ & DIEC2 en línia \\
\hline Descripción breve & Diccionario del Institut d'Estudis Catalans \\
\hline Enlace & $\begin{array}{l}\text { https://play.google.com/store/apps/details? } \\
\text { id=com.iec.diccionarionline\&hl=ca }\end{array}$ \\
\hline Sistema(s) & Android \\
\hline
\end{tabular}




\begin{tabular}{|l|l|}
\hline operativo(s) & \\
\hline Precio/Licencia & Gratuita \\
\hline Versión actual & 1.0 .1 \\
\hline Observaciones & \\
\hline Colaborador & \\
\hline
\end{tabular}

\begin{tabular}{|l|l|}
\hline $\begin{array}{l}\text { Nombre de la } \\
\text { herramienta }\end{array}$ & DRAE \\
\hline Descripción breve & Diccionario monolingüe de español, de la Real Academia Española \\
\hline Enlace & $\begin{array}{l}\text { https://itunes.apple.com/us/app/dle/id1011116985?/=es } \\
\text { https://play.google.com/store/apps/details?id=es.rae.dle }\end{array}$ \\
\hline $\begin{array}{l}\text { Sistema(s) } \\
\text { operativo(s) }\end{array}$ & $\begin{array}{l}\text { iOS } \\
\text { Android }\end{array}$ \\
\hline Precio/Licencia & GacOS \\
\hline Versión actual & $\begin{array}{l}1.0 .1 \text { (iOS) } \\
1.2 .1 \text { (Android) }\end{array}$ \\
\hline Observaciones & \\
\hline Colaborador & $\begin{array}{l}\text { Daniel Salinero } \\
\text { TheWriteTranslator@verizon.net } \\
\text { TheWriteTranslator.com }\end{array}$ \\
\hline
\end{tabular}

\begin{tabular}{|l|l|}
\hline $\begin{array}{l}\text { Nombre de la } \\
\text { herramienta }\end{array}$ & Linguee \\
\hline Descripción breve & $\begin{array}{l}\text { Diccionario multilingüe y corpus de traducciones alineadas con sus } \\
\text { textos de origen }\end{array}$ \\
\hline Enlace & www.linguee.com \\
\hline $\begin{array}{l}\text { Sistema(s) } \\
\text { operativo(s) }\end{array}$ & $\begin{array}{l}\text { iOS } \\
\text { Android }\end{array}$ \\
\hline Precio/Licencia & Gratuita \\
\hline Versión actual & $\begin{array}{l}2.3 .1 \text { (iOS) } \\
1.2 .4 \text { (Android) }\end{array}$ \\
\hline Observaciones & \\
\hline Colaborador & $\begin{array}{l}\text { Oscar Muñoz Pacheco } \\
\text { oscar.traductorjurado@gmail.com } \\
\text { UVIC }\end{array}$ \\
\hline
\end{tabular}

\begin{tabular}{|l|l|}
\hline $\begin{array}{l}\text { Nombre de la } \\
\text { herramienta }\end{array}$ & Merriam-Webster \\
\hline Descripción breve & Diccionario monolingüe de inglés \\
\hline Enlace & https://www.merriam-webster.com/apps \\
\hline $\begin{array}{l}\text { Sistema(s) } \\
\text { operativo(s) }\end{array}$ & $\begin{array}{l}\text { iOS } \\
\text { Android }\end{array}$ \\
& Multiplataforma (web) \\
\hline
\end{tabular}




\begin{tabular}{|l|l|}
\hline Precio/Licencia & Gratuita \\
\hline Versión actual & 4.0 .1 \\
\hline Observaciones & \\
\hline Colaborador & $\begin{array}{l}\text { Daniel Salinero } \\
\text { TheWriteTranslator@verizon.net } \\
\text { TheWriteTranslator.com }\end{array}$ \\
\hline
\end{tabular}

\begin{tabular}{|l|l|}
\hline $\begin{array}{l}\text { Nombre de la } \\
\text { herramienta }\end{array}$ & Pons \\
\hline Descripción breve & Diccionario de alemán \\
\hline Enlace & $\begin{array}{l}\text { https://itunes.apple.com/us/app/pons-online-translator- } \\
\text { free/id577741918? } \mathrm{mt}=8 \\
\text { https://play.google.com/store/apps/details? } \\
\text { id=com.pons.onlinedictionary\&hl=es }\end{array}$ \\
\hline $\begin{array}{l}\text { Sistema(s) } \\
\text { operativo(s) }\end{array}$ & $\begin{array}{l}\text { OOS } \\
\text { Android }\end{array}$ \\
\hline Precio/Licencia & Gratuita \\
\hline Versión actual & 3.0.6 (iOS) \\
\hline Observaciones & \\
\hline Colaborador & $\begin{array}{l}\text { Cristina Plaza Lara } \\
\text { cplaza@uma.es }\end{array}$ \\
Universidad de Málaga \\
\hline
\end{tabular}

\begin{tabular}{|l|l|}
\hline $\begin{array}{l}\text { Nombre de la } \\
\text { herramienta }\end{array}$ & WordReference \\
\hline Descripción breve & Diccionarios monolingües y bilingües \\
\hline Enlace & http://www.wordreference.com/es/translation.asp?tranword=app \\
\hline $\begin{array}{l}\text { Sistema(s) } \\
\text { operativo(s) }\end{array}$ & $\begin{array}{l}\text { IOS } \\
\text { Android } \\
\text { Multiplataforma (web) }\end{array}$ \\
\hline Precio/Licencia & Gratuita \\
\hline Versión actual & 5.0 .9 \\
\hline Observaciones & \\
\hline Colaborador & $\begin{array}{l}\text { Daniel Salinero } \\
\text { TheWriteTranslator@verizon.net } \\
\text { TheWriteTranslator.com }\end{array}$ \\
\hline
\end{tabular}

\section{Gestión de la terminología}

\begin{tabular}{|l|l|}
\hline $\begin{array}{l}\text { Nombre de la } \\
\text { herramienta }\end{array}$ & Glossary Assistant \\
\hline Descripción breve & $\begin{array}{l}\text { Herramienta intuitiva con múltiples alfabetos. Permite la creación de } \\
\text { glosarios de gran tamaño. Además, se pueden importar glosarios } \\
\text { existentes en otros formatos. Se basa en el toque con el dedo. }\end{array}$ \\
\hline Enlace & $\begin{array}{l}\text { https://play.google.com/store/apps/details? } \\
\text { id=conference.interpeter.glossaryassistant\&hl=es }\end{array}$ \\
\hline Sistema(s) & Android \\
\hline
\end{tabular}




\begin{tabular}{|l|l|}
\hline operativo(s) & \\
\hline Precio/Licencia & Gratuita \\
\hline Versión actual & \\
\hline Observaciones & Desarrollada para intérpretes de conferencias \\
\hline Colaborador & $\begin{array}{l}\text { María Teresa Ortego Antón } \\
\text { tortego@lesp.uva.es }\end{array}$ \\
Universidad de Valladolid \\
\hline
\end{tabular}

\begin{tabular}{|l|l|}
\hline $\begin{array}{l}\text { Nombre de la } \\
\text { herramienta }\end{array}$ & Interplex \\
\hline Descripción breve & $\begin{array}{l}\text { Programa de gestión de glosarios que puede utilizarse de forma fácil } \\
\text { y rápida mientras se interpreta. }\end{array}$ \\
\hline Enlace & http://www.fourwillows.com/interplex.html \\
\hline $\begin{array}{l}\text { Sistema(s) } \\
\text { operativo(s) }\end{array}$ & $\begin{array}{l}\text { OSS } \\
\text { Windows }\end{array}$ \\
\hline Precio/Licencia & Gratuita \\
\hline Versión actual & 1.60 \\
\hline Observaciones & $\begin{array}{l}\text { La versión para iOS es más simple y se denomina Interplex Lite para } \\
\text { iPhone e Interplex HD para iPad. }\end{array}$ \\
\hline Colaborador & $\begin{array}{l}\text { María Teresa Ortego Antón } \\
\text { tortego@lesp.uva.es } \\
\text { Universidad de Valladolid } \\
\text { Rocío del Pozo Caamaño } \\
\text { rociodelpozo@gmail.com } \\
\text { Traductora e intérprete autónoma }\end{array}$ \\
\hline
\end{tabular}

\begin{tabular}{|l|l|}
\hline $\begin{array}{l}\text { Nombre de la } \\
\text { herramienta }\end{array}$ & InterpretBank \\
\hline Descripción breve & $\begin{array}{l}\text { Herramienta de gestión terminológica diseñada para intérpretes. } \\
\text { Permite compartir glosarios con colegas y sincronizar varios } \\
\text { dispositivos. Tiene funciones de importación y exportación, y } \\
\text { traducción automática. }\end{array}$ \\
\hline Enlace & $\begin{array}{l}\text { http://www.interpretbank.de/ } \\
\text { https://play.google.com/store/apps/details?id=ryap.inter.pretbank }\end{array}$ \\
\hline $\begin{array}{l}\text { Sistema(s) } \\
\text { operativo(s) }\end{array}$ & $\begin{array}{l}\text { Android } \\
\text { Windows }\end{array}$ \\
\hline Precio/Licencia & $\begin{array}{l}\text { Gratuita (Android) } \\
99 € \text { (Windows) }\end{array}$ \\
\hline Versión actual & 1.4 \\
\hline Observaciones & $\begin{array}{l}\text { Pronto disponible para MacOS, donde ya se puede usar por medio } \\
\text { de Crossover. }\end{array}$ \\
\hline Colaborador & $\begin{array}{l}\text { María Teresa Ortego Antón } \\
\text { tortego@lesp.uva.es } \\
\text { Universidad de Valladolid } \\
\text { Rocío del Pozo Caamaño } \\
\text { rociodelpozo@gmail.com } \\
\text { Traductora e intérprete autónoma }\end{array}$ \\
\hline
\end{tabular}




\begin{tabular}{|l|l|}
\hline $\begin{array}{l}\text { Nombre de la } \\
\text { herramienta }\end{array}$ & Interpreters' Help \\
\hline Descripción breve & $\begin{array}{l}\text { Herramienta de gestión terminológica que se organiza en glosarios } \\
\text { con funciones de edición }\end{array}$ \\
\hline Enlace & https://interpretershelp.com/ \\
\hline $\begin{array}{l}\text { Sistema(s) } \\
\text { operativo(s) }\end{array}$ & Multiplataforma (web) \\
\hline Precio/Licencia & $19,99 € /$ mes \\
\hline Versión actual & 2016 \\
\hline Observaciones & Existe versión gratuita con funciones limitadas \\
\hline Colaborador & $\begin{array}{l}\text { María Teresa Ortego Antón } \\
\text { tortego@lesp.uva.es } \\
\text { Universidad de Valladolid }\end{array}$ \\
\hline
\end{tabular}

\begin{tabular}{|l|l|}
\hline $\begin{array}{l}\text { Nombre de la } \\
\text { herramienta }\end{array}$ & LookUp \\
\hline Descripción breve & $\begin{array}{l}\text { Herramienta de gestión terminológica con una rápida función de } \\
\text { búsqueda }\end{array}$ \\
\hline Enlace & http://www.dog-gmbh.de/software-products/lookup.html?L=1 \\
\hline $\begin{array}{l}\text { Sistema(s) } \\
\text { operativo(s) }\end{array}$ & Multiplataforma (web) \\
\hline Precio/Licencia & $5900 €$ para cinco usuarios \\
\hline Versión actual & LookUp 7 \\
\hline Observaciones & $\begin{array}{l}\text { María Teresa Ortego Antón } \\
\text { tortego@lesp.uva.es } \\
\text { Universidad de Valladolid }\end{array}$ \\
\hline Colaborador &
\end{tabular}

\begin{tabular}{|l|l|}
\hline $\begin{array}{l}\text { Nombre de la } \\
\text { herramienta }\end{array}$ & Memento Database \\
\hline Descripción breve & $\begin{array}{l}\text { Base de datos que se puede usar para crear glosarios propios. Se } \\
\text { puede sincronizar con Google Sheets, y desde Google Sheets es } \\
\text { posible modificar la información que tenemos en Memento. Ofrece la } \\
\text { posibilidad de trabajar en equipo mediante Memento Cloud. }\end{array}$ \\
\hline Enlace & $\begin{array}{l}\text { http://mementodatabase.com } \\
\text { https://play.google.com/store/apps/details? } \\
\text { id=com.luckydroid.droidbase\&referrer=utm_source\%3Dappsite }\end{array}$ \\
\hline $\begin{array}{l}\text { Sistema(s) } \\
\text { operativo(s) }\end{array}$ & Android \\
\hline Precio/Licencia & Gratuita \\
\hline Versión actual & 4.1 .0 \\
\hline Observaciones & \\
\hline Colaborador & $\begin{array}{l}\text { Rocío del Pozo Caamaño } \\
\text { rociodelpozo@gmail.com } \\
\text { Traductora e intérprete autónoma }\end{array}$ \\
\hline
\end{tabular}




\begin{tabular}{|l|l|}
\hline $\begin{array}{l}\text { Nombre de la } \\
\text { herramienta }\end{array}$ & Tap Forms \\
\hline Descripción breve & Base de datos que se puede editar para crear un glosario multilingüe \\
\hline Enlace & www.tapforms.com \\
\hline $\begin{array}{l}\text { Sistema(s) } \\
\text { operativo(s) }\end{array}$ & iOS \\
\hline Precio/Licencia & $16,99 \$$ \\
\hline Versión actual & 5.0 .8 \\
\hline Observaciones & Se puede sincronizar fácilmente con iCloud o Dropbox \\
\hline Colaborador & $\begin{array}{l}\text { Rocío del Pozo Caamaño } \\
\text { rociodelpozo@gmail.com }\end{array}$ \\
& Traductora e intérprete autónoma \\
\hline
\end{tabular}

\begin{tabular}{|l|l|}
\hline $\begin{array}{l}\text { Nombre de la } \\
\text { herramienta }\end{array}$ & Terminus \\
\hline Descripción breve & $\begin{array}{l}\text { Sistema de gestión terminológica multilingüe diseñado por } \\
\text { intérpretes para intérpretes }\end{array}$ \\
\hline Enlace & http://terminus.iula.upf.edu/cgi-bin/terminus2.0/terminus.pl \\
\hline $\begin{array}{l}\text { Sistema(s) } \\
\text { operativo(s) }\end{array}$ & Multiplataforma (web) \\
\hline Precio/Licencia & 2.0 \\
\hline Versión actual & También permite gestionar corpus \\
\hline Observaciones & $\begin{array}{l}\text { María Teresa Ortego Antón } \\
\text { tortego@lesp.uva.es }\end{array}$ \\
\hline Colaborador & Universidad de Valladolid \\
\hline
\end{tabular}

\section{Gestión de tareas y proyectos}

\begin{tabular}{|l|l|}
\hline $\begin{array}{l}\text { Nombre de la } \\
\text { herramienta }\end{array}$ & Todoist \\
\hline Descripción breve & Herramienta de gestión de listas de tareas y productividad \\
\hline Enlace & http://todoist.com \\
\hline $\begin{array}{l}\text { Sistema(s) } \\
\text { operativo(s) }\end{array}$ & $\begin{array}{l}\text { OS } \\
\text { Android } \\
\text { Windows Phone } \\
\text { Multiplataforma (web) }\end{array}$ \\
\hline Precio/Licencia & $\begin{array}{l}\text { Gratuita (salvo sus versiones Premium, a 28,99 €/año, y Business, a } \\
28,99 € / \text { usuario) }\end{array}$ \\
\hline Versión actual & $\begin{array}{l}11.2 .8 \text { (iOS) } \\
11.1 .2 \text { (Android) } \\
1.1 .80 .0 \text { (Windows Phone) }\end{array}$ \\
\hline Observaciones & $\begin{array}{l}\text { Todoist es una herramienta ideal para desglosar tareas y trabajar en } \\
\text { equipo. También se puede usar a título individual, ya que también es } \\
\text { genial para organizar tu tiempo y para desglosar proyectos en } \\
\text { subtareas para llevarlos a cabo de una forma más ordenada y }\end{array}$ \\
\hline
\end{tabular}




\begin{tabular}{|l|l|}
\hline & $\begin{array}{l}\text { productiva. Además, es compatible con diferentes sistemas } \\
\text { operativos, de forma que el usuario puede acceder a sus listas } \\
\text { desde varios dispositivos de forma simultánea, lo que la convierte en } \\
\text { una de las mejores herramientas de productividad del momento. }\end{array}$ \\
\hline Colaborador & $\begin{array}{l}\text { Rafael López Sánchez } \\
\text { rafael@rafaellopezsanchez.com } \\
\text { Universidad de Granada }\end{array}$ \\
\hline
\end{tabular}

\begin{tabular}{|c|c|}
\hline $\begin{array}{l}\text { Nombre de la } \\
\text { herramienta }\end{array}$ & Toggl \\
\hline Descripción breve & $\begin{array}{l}\text { Toggl permite controlar el tiempo que dedicamos a todas las tareas } \\
\text { que realizamos en nuestro día a día. Pueden ser de traducción o de } \\
\text { cualquier otra cosa, y de hecho se pueden configurar varios } \\
\text { "espacios de trabajo" (por ejemplo, "Personal", "Traducciones", } \\
\text { "Clases"). Aunque hay que ser disciplinado y metódico, de esta } \\
\text { manera se puede controlar fácilmente el tiempo que le dedicamos a } \\
\text { cada tarea para ver si somos productivos y si nuestro trabajo es } \\
\text { rentable, ya que todos estos datos te permiten saber el precio por } \\
\text { hora de tu trabajo en función de tus resultados. Asimismo, sus } \\
\text { completas gráficas te ayudan a saber qué aspectos debes mejorar } \\
\text { para ser aún más productivo (o ver si realmente un cliente o tarea te } \\
\text { es rentable o no en términos de tiempo/dinero). }\end{array}$ \\
\hline Enlace & https://toggl.com/ \\
\hline $\begin{array}{l}\text { Sistema(s) } \\
\text { operativo(s) }\end{array}$ & $\begin{array}{l}\text { iOS } \\
\text { Android } \\
\text { Multiplataforma (web) }\end{array}$ \\
\hline Precio/Licencia & Gratis / 10 \$ al mes (versión Pro) / 20 \$ al mes (versión Pro Plus) \\
\hline Versión actual & $\begin{array}{l}\text { 7.2.3 (iOS) } \\
\text { 8.1.5 (Android) }\end{array}$ \\
\hline \multicolumn{2}{|l|}{ Observaciones } \\
\hline Colaborador & $\begin{array}{l}\text { Pablo Muñoz Sánchez } \\
\text { pmstrad@gmail.com } \\
\text { Universitat Autònoma de Barcelona }\end{array}$ \\
\hline
\end{tabular}

\begin{tabular}{|l|l|}
\hline $\begin{array}{l}\text { Nombre de la } \\
\text { herramienta }\end{array}$ & Trello \\
\hline Descripción breve & $\begin{array}{l}\text { Aplicación para organizar proyectos y tareas. Aunque no está } \\
\text { pensada exclusivamente para traductores, es una herramienta muy } \\
\text { útil de organización y gestión de proyectos compartidos. }\end{array}$ \\
\hline Enlace & https://trello.com/ \\
\hline $\begin{array}{l}\text { Sistema(s) } \\
\text { operativo(s) }\end{array}$ & $\begin{array}{l}\text { iOS } \\
\text { Android } \\
\text { Multiplataforma (web) }\end{array}$ \\
\hline Precio/Licencia & Gratuita \\
\hline Versión actual & $\begin{array}{l}3.4 .7 \text { (iOS) } \\
3.8 .4 .2152 \text { (Android) }\end{array}$ \\
\hline Observaciones & $\begin{array}{l}\text { Permite visualizar todo un proyecto con tan solo echar un vistazo al } \\
\text { tablero; los cambios se actualizan en tiempo real. No es necesario } \\
\text { realizar configuraciones complicadas. Es compatible con Chrome, }\end{array}$ \\
\hline
\end{tabular}




\begin{tabular}{|l|l|}
\hline & $\begin{array}{l}\text { Firefox, Safari (6+) e Internet Explorer (10+). También admite la } \\
\text { mayoría de los navegadores para móvil, como Safari en iOS 8, el } \\
\text { navegador de Android 4.0+ e Internet Explorer en Windows Phone. }\end{array}$ \\
\hline Colaborador & $\begin{array}{l}\text { Susana Álvarez-Álvarez } \\
\text { susanalv@lesp.uva.es } \\
\text { Facultad de Traducción e Interpretación (Universidad de Valladolid) }\end{array}$ \\
\hline
\end{tabular}

\section{Toma de notas}

\begin{tabular}{|l|l|}
\hline $\begin{array}{l}\text { Nombre de la } \\
\text { herramienta }\end{array}$ & Evernote \\
\hline Descripción breve & $\begin{array}{l}\text { Herramienta para organizar la información. Se pueden escribir notas, } \\
\text { hacer listas, digitalizar distintos tipos de documentos físicos, guardar } \\
\text { páginas web y organizarlas. Permite sincronizar toda la información. }\end{array}$ \\
\hline Enlace & https://evernote.com \\
\hline $\begin{array}{l}\text { Sistema(s) } \\
\text { operativo(s) }\end{array}$ & $\begin{array}{l}\text { OS } \\
\text { Android }\end{array}$ \\
\hline Precio/Licencia & Gratuita \\
\hline Versión actual & $\begin{array}{l}7.18 .1 \text { (iOS) } \\
23 \text { de diciembre de 2016 (Android) }\end{array}$ \\
\hline Observaciones & $\begin{array}{l}\text { Rocío del Pozo Caamaño } \\
\text { rociodelpozo@gmail.com } \\
\text { Traductora e intérprete autónoma }\end{array}$ \\
\hline Colaborador &
\end{tabular}

\begin{tabular}{|l|l|}
\hline $\begin{array}{l}\text { Nombre de la } \\
\text { herramienta }\end{array}$ & MyScript Memo \\
\hline Descripción breve & Aplicación para la toma de notas, más sencilla que otras. \\
\hline Enlace & http://myscript.com/myscript-memo/ \\
\hline $\begin{array}{l}\text { Sistema(s) } \\
\text { operativo(s) }\end{array}$ & iOS \\
\hline Precio/Licencia & Gratuita \\
\hline Versión actual & 3.0 .0 \\
\hline Observaciones & $\begin{array}{l}\text { Rocío del Pozo Caamaño } \\
\text { rociodelpozo@gmail.com } \\
\text { Traductora e intérprete autónoma }\end{array}$ \\
\hline Colaborador &
\end{tabular}

\begin{tabular}{|l|l|}
\hline $\begin{array}{l}\text { Nombre de la } \\
\text { herramienta }\end{array}$ & Notability \\
\hline Descripción breve & $\begin{array}{l}\text { Aplicación para la toma de notas. Incluye la posibilidad de grabar el } \\
\text { audio al mismo tiempo. Además, el audio grabado se puede } \\
\text { sincronizar con las notas. }\end{array}$ \\
\hline Enlace & http://gingerlabs.com/ \\
\hline
\end{tabular}




\begin{tabular}{|l|l|}
\hline $\begin{array}{l}\text { Sistema(s) } \\
\text { operativo(s) }\end{array}$ & iOS \\
\hline Precio/Licencia & $6,99 €$ \\
\hline Versión actual & 6.4 .2 \\
\hline Observaciones & $\begin{array}{l}\text { Rocío del Pozo Caamaño } \\
\text { rociodelpozo@gmail.com } \\
\text { Traductora e intérprete autónoma }\end{array}$ \\
\hline Colaborador
\end{tabular}

\begin{tabular}{|l|l|}
\hline $\begin{array}{l}\text { Nombre de la } \\
\text { herramienta }\end{array}$ & Penultimate \\
\hline Descripción breve & $\begin{array}{l}\text { Aplicación para la toma de notas. Ofrece varias opciones como color, } \\
\text { grosor del trazo, forma del papel, postura horizontal o vertical del } \\
\text { papel, se puede escribir con el dedo o con un stylus. }\end{array}$ \\
\hline Enlace & https://evernote.com/int//es/penultimate/ \\
\hline $\begin{array}{l}\text { Sistema(s) } \\
\text { operativo(s) }\end{array}$ & OS \\
\hline Precio/Licencia & Gratuita \\
\hline Versión actual & 6.2 .1 \\
\hline Observaciones & \\
\hline Colaborador & $\begin{array}{l}\text { Rocío del Pozo Caamaño } \\
\text { rociodelpozo@gmail.com }\end{array}$ \\
\hline
\end{tabular}

\section{Interacción con dispositivos}

\begin{tabular}{|l|l|}
\hline $\begin{array}{l}\text { Nombre de la } \\
\text { herramienta }\end{array}$ & Dragon Dictation \\
\hline Descripción breve & Reconocimiento de voz \\
\hline Enlace & https://itunes.apple.com/us/app/dragon-dictation/id341446764?mt=8 \\
\hline $\begin{array}{l}\text { Sistema(s) } \\
\text { operativo(s) }\end{array}$ & iOS \\
\hline Precio/Licencia & Gratuita \\
\hline Versión actual & 2.0 .28 \\
\hline Observaciones & $\begin{array}{l}\text { La precisión del reconocimiento de voz es muy alta, en comparación } \\
\text { con otras apps (con la reserva de que lo he probado siempre para el } \\
\text { ruso), por lo tanto puede ser utilizado para crear textos } \\
\text { aumentando/manteniendo la productividad, en varias circunstancias. }\end{array}$ \\
\hline Colaborador & $\begin{array}{l}\text { Ekaterina Guerbek } \\
\text { ekat.guer@gmail.com } \\
\text { Traductora autónoma }\end{array}$ \\
\hline
\end{tabular}

\begin{tabular}{|l|l|}
\hline $\begin{array}{l}\text { Nombre de la } \\
\text { herramienta }\end{array}$ & Varias \\
\hline Descripción breve & $\begin{array}{l}\text { Teclados virtuales con funciones predictivas (recopilación por } \\
\text { Softcatalà) }\end{array}$ \\
\hline
\end{tabular}




\begin{tabular}{|l|l|}
\hline Enlace & $\begin{array}{l}\text { https://www.softcatala.org/ordinadors-i-mobils-en- } \\
\text { catala/tutorials/configurar-android-en-catala/ }\end{array}$ \\
\hline $\begin{array}{l}\text { Sistema(s) } \\
\text { operativo(s) }\end{array}$ & Android \\
\hline Precio/Licencia & Varias \\
\hline Versión actual & Varias \\
\hline Observaciones & \\
\hline Colaborador & \\
\hline
\end{tabular}

\begin{tabular}{|l|l|}
\hline $\begin{array}{l}\text { Nombre de la } \\
\text { herramienta }\end{array}$ & Swype \\
\hline Descripción breve & Teclado virtual rápido con reconocimiento de voz (Dragon) \\
\hline Enlace & www.swype.com \\
\hline $\begin{array}{l}\text { Sistema(s) } \\
\text { operativo(s) }\end{array}$ & $\begin{array}{l}\text { iOS } \\
\text { Android } \\
\text { Windows Phone } \\
\text { Windows 7 }\end{array}$ \\
\hline Precio/Licencia & $\begin{array}{l}\text { OOS: } 0,99 € \\
\text { Android: } 1,13 €\end{array}$ \\
\hline Versión actual & $\begin{array}{l}1.6 .3 \text { (iOS) } \\
19 \text { de julio de 2016 (Android) }\end{array}$ \\
\hline Observaciones & $\begin{array}{l}\text { Léanse las condiciones de uso antes de utilizarlo para garantizar la } \\
\text { protección de los datos. }\end{array}$ \\
\hline Colaborador & \multicolumn{2}{|l}{} \\
\hline
\end{tabular}

\section{Edición de traducciones}

\begin{tabular}{|l|l|}
\hline $\begin{array}{l}\text { Nombre de la } \\
\text { herramienta }\end{array}$ & LanguageTool \\
\hline Descripción breve & $\begin{array}{l}\text { Corrector ortotipográfico, gramatical y de estilo para unas treinta } \\
\text { lenguas. }\end{array}$ \\
\hline Enlace & https://play.google.com/store/apps/details?id=org.softcatala.corrector \\
\hline $\begin{array}{l}\text { Sistema(s) } \\
\text { operativo(s) }\end{array}$ & Android \\
\hline Precio/Licencia & Gratuita/libre \\
\hline Versión actual & 1.0 .6 \\
\hline Observaciones & \\
\hline Colaborador & \\
\hline
\end{tabular}

\begin{tabular}{|l|l|}
\hline $\begin{array}{l}\text { Nombre de la } \\
\text { herramienta }\end{array}$ & Loco - strings to XML converter \\
\hline Descripción breve & $\begin{array}{l}\text { Conversor de formatos. Puede convertir entre strings (para iOS) y } \\
\text { XML (para Android), y permite exportar a XLIFF. }\end{array}$ \\
\hline Enlace & https://localise.biz/free/converter/ios-to-android \\
\hline
\end{tabular}




\begin{tabular}{|l|l|}
\hline $\begin{array}{l}\text { Sistema(s) } \\
\text { operativo(s) }\end{array}$ & Multiplataforma (web) \\
\hline Precio/Licencia & Gratuita \\
\hline Versión actual & \\
\hline Observaciones & \\
\hline Colaborador & \\
\hline
\end{tabular}

\begin{tabular}{|l|l|}
\hline $\begin{array}{l}\text { Nombre de la } \\
\text { herramienta }\end{array}$ & MateCat \\
\hline Descripción breve & Sistema de gestión y de edición de traducciones en la nube \\
\hline Enlace & www.matecat.com \\
\hline $\begin{array}{l}\text { Sistema(s) } \\
\text { operativo(s) }\end{array}$ & Multiplataforma (web) \\
\hline Precio/Licencia & Gratuita/LGPL \\
\hline Versión actual & $\begin{array}{l}\text { Actualmente solo funciona con Chrome/Chromium, por lo que es } \\
\text { accesible desde cualquier dispositivo que permita instalar este } \\
\text { navegador. } \\
\text { Incluye funciones de análisis de la productividad, conexión de } \\
\text { motores de traducción automática y filtros para formatos de } \\
\text { localización de aplicaciones móviles. }\end{array}$ \\
\hline Observaciones \\
\hline Colaborador & \\
\hline
\end{tabular}

\section{Traducción automática y postedición}

\begin{tabular}{|l|l|}
\hline $\begin{array}{l}\text { Nombre de la } \\
\text { herramienta }\end{array}$ & Apertium offline translator \\
\hline Descripción breve & $\begin{array}{l}\text { Motor de traducción automática basada en reglas para unas } \\
\text { cuarenta lenguas, muchas de ellas minorizadas. }\end{array}$ \\
\hline Enlace & https://play.google.com/store/apps/details?id=org.apertium.android \\
\hline $\begin{array}{l}\text { Sistema(s) } \\
\text { operativo(s) }\end{array}$ & Android \\
\hline Precio/Licencia & Gratuita/Libre \\
\hline Versión actual & 1.02 \\
\hline Observaciones & \\
\hline Colaborador & \\
\hline
\end{tabular}

\begin{tabular}{|l|l|}
\hline $\begin{array}{l}\text { Nombre de la } \\
\text { herramienta }\end{array}$ & Babylon \\
\hline Descripción breve & Traducción automática \\
\hline Enlace & www.babylon-software.com \\
\hline $\begin{array}{l}\text { Sistema(s) } \\
\text { operativo(s) }\end{array}$ & $\begin{array}{l}\text { iOS } \\
\text { Android } \\
\text { Windows Phone } \\
\end{array}$ Blackberry \\
& Kindle \\
\hline
\end{tabular}




\begin{tabular}{|l|l|}
\hline & $\begin{array}{l}\text { Multiplataforma (web) } \\
\text { Windows }\end{array}$ \\
\hline Precio/Licencia & Gratuita \\
\hline Versión actual & Varias \\
\hline Observaciones & $\begin{array}{l}\text { Daniel Salinero } \\
\text { TheWriteTranslator@verizon.net } \\
\text { TheWriteTranslator.com }\end{array}$ \\
\hline Colaborador
\end{tabular}

\begin{tabular}{|l|l|}
\hline $\begin{array}{l}\text { Nombre de la } \\
\text { herramienta }\end{array}$ & Kanjingo \\
\hline Descripción breve & $\begin{array}{l}\text { Aplicación para la posedición móvil. Su interfaz permite reordenar y } \\
\text { modificar palabras mediante gestos y voz. }\end{array}$ \\
\hline Enlace & www.kanjingo.com \\
\hline $\begin{array}{l}\text { Sistema(s) } \\
\text { operativo(s) }\end{array}$ & OS \\
\hline Precio/Licencia & N/A \\
\hline Versión actual & 0.6 \\
\hline Observaciones & $\begin{array}{l}\text { Actualmente no está disponible como producto comercial, pero su } \\
\text { desarrollo va a proseguir durante 2017. }\end{array}$ \\
\hline Colaborador & $\begin{array}{l}\text { Joss Moorkens } \\
\text { joss.moorkens@dcu.ie } \\
\text { Dublin City University }\end{array}$ \\
\hline
\end{tabular}

\begin{tabular}{|l|l|}
\hline $\begin{array}{l}\text { Nombre de la } \\
\text { herramienta }\end{array}$ & Mitzuli \\
\hline Descripción breve & $\begin{array}{l}\text { Aplicación que usa varios motores de traducción automática } \\
\text { (Apertium, Matxin, Abu-MaTran). }\end{array}$ \\
\hline Enlace & www.mitzuli.com \\
\hline $\begin{array}{l}\text { Sistema(s) } \\
\text { operativo(s) }\end{array}$ & Android \\
\hline Precio/Licencia & Gratuita/Libre \\
\hline Versión actual & $\begin{array}{l}\text { Incluye reconocimiento de voz (mediante el micrófono), } \\
\text { reconocimiento de imágenes (mediante la cámara) y lectura de texto } \\
\text { (mediante el altavoz). }\end{array}$ \\
\hline Observaciones & \\
\hline Colaborador &
\end{tabular}

\begin{tabular}{|l|l|}
\hline $\begin{array}{l}\text { Nombre de la } \\
\text { herramienta }\end{array}$ & Traductor de Softcatalà \\
\hline Descripción breve & Traductor automático basado en Apertium \\
\hline Enlace & https://play.google.com/store/apps/details?id=org.softcatala.traductor \\
\hline $\begin{array}{l}\text { Sistema(s) } \\
\text { operativo(s) }\end{array}$ & Android \\
\hline Precio/Licencia & Gratuita \\
\hline Versión actual & \\
\hline
\end{tabular}




\begin{tabular}{|l|l|}
\hline Observaciones & Síntesis de voz \\
\hline Colaborador & \\
\hline
\end{tabular}

\section{Agregación de contenidos}

\begin{tabular}{|l|l|}
\hline $\begin{array}{l}\text { Nombre de la } \\
\text { herramienta }\end{array}$ & Feedly \\
\hline Descripción breve & $\begin{array}{l}\text { Lector de noticias. La información se puede compartir en las redes } \\
\text { sociales, así como en OneNote 365 y Evernote Business. }\end{array}$ \\
\hline Enlace & https://feedly.com/i/welcome \\
\hline $\begin{array}{l}\text { Sistema(s) } \\
\text { operativo(s) }\end{array}$ & $\begin{array}{l}\text { iOS } \\
\text { Android } \\
\text { Kindle }\end{array}$ \\
\hline Precio/Licencia & Gratuita \\
\hline Versión actual & $\begin{array}{l}36.0 .0 \text { (iOS) } \\
34.0 .0 \text { (Android) }\end{array}$ \\
\hline Observaciones & Su diseño es simple y se puede personalizar \\
\hline Colaborador & $\begin{array}{l}\text { Rocío del Pozo Caamaño } \\
\text { rociodelpozo@gmail.com }\end{array}$ \\
\cline { 2 - 2 } & Traductora e intérprete autónoma \\
\hline
\end{tabular}

\begin{tabular}{|l|l|}
\hline $\begin{array}{l}\text { Nombre de la } \\
\text { herramienta }\end{array}$ & Flipboard \\
\hline Descripción breve & $\begin{array}{l}\text { Lector de noticias. Permite ver las novedades de noticias, periódicos, } \\
\text { redes sociales como Twitter, Facebook, así como organizar y } \\
\text { compartir la información. }\end{array}$ \\
\hline Enlace & https://about.flipboard.com/tutorials/ \\
\hline $\begin{array}{l}\text { Sistema(s) } \\
\text { operativo(s) }\end{array}$ & $\begin{array}{l}\text { OSS } \\
\text { Android } \\
\text { Windows Phone } \\
\text { Windows }\end{array}$ \\
\hline Precio/Licencia & Gratuita \\
\hline Versión actual & $\begin{array}{l}\text { 3.3.32 (iOS) } \\
28 \text { de octubre de 2016 (Android) }\end{array}$ \\
\hline Observaciones & $\begin{array}{l}\text { Rocío del Pozo Caamaño } \\
\text { rociodelpozo@gmail.com } \\
\text { Traductora e intérprete autónoma }\end{array}$ \\
\hline Colaborador & \\
\hline
\end{tabular}

\begin{tabular}{|l|l|}
\hline $\begin{array}{l}\text { Nombre de la } \\
\text { herramienta }\end{array}$ & Pocket \\
\hline Descripción breve & $\begin{array}{l}\text { Lector de noticias. Permite guardar artículos para leerlos más tarde } \\
\text { desde cualquier sitio: Facebook, Twitter, Flipboard, Feedly, Safari, el } \\
\text { correo electrónico, el PC. Una vez guardados, se pueden leer sin } \\
\text { estar en línea. }\end{array}$ \\
\hline Enlace & $\begin{array}{l}\text { www.getpocket.com } \\
\text { https://itunes.apple.com/app/read-it-later-pro/id309601447?mt=8 }\end{array}$ \\
\hline
\end{tabular}




\begin{tabular}{|l|l|}
\hline & https://play.google.com/store/apps/details? \\
& id=com.ideashower.readitlater.pro \\
\hline $\begin{array}{l}\text { Sistema(s) } \\
\text { operativo(s) }\end{array}$ & iOS \\
& Android \\
& Multiplataforma (web) \\
\hline Precio/Licencia & Gratuita \\
\hline Versión actual & 6.4 .8 (iOS) \\
& 6.4 .7 .0 (Android) \\
\hline Observaciones & \\
\hline Colaborador & Rocío del Pozo Caamaño \\
& rociodelpozo@gmail.com \\
\cline { 2 - 2 } & Traductora e intérprete autónoma \\
\hline
\end{tabular}

\begin{tabular}{|c|c|}
\hline $\begin{array}{l}\text { Nombre de la } \\
\text { herramienta }\end{array}$ & Pocket Casts \\
\hline Descripción breve & Gestor de suscripciones a podcasts \\
\hline Enlace & $\begin{array}{l}\text { https://play.pocketcasts.com/ } \\
\text { https://itunes.apple.com/es/app/pocket-casts/id414834813?mt=8 } \\
\text { https://play.google.com/store/apps/details? } \\
\text { id=au.com.shiftyjelly.pocketcasts\&hl=es } \\
\text { https://www.microsoft.com/es-es/store/p/pocket-casts/9nblggh3majh }\end{array}$ \\
\hline $\begin{array}{l}\text { Sistema(s) } \\
\text { operativo(s) }\end{array}$ & $\begin{array}{l}\text { iOS } \\
\text { Android } \\
\text { Windows Phone } \\
\end{array}$ \\
\hline Precio/Licencia & $\begin{array}{l}3,99 €(\text { iOS }) \\
2,99 € \text { (Android) } \\
2,99 € \text { (Windows Phone) }\end{array}$ \\
\hline Versión actual & $\begin{array}{l}6.4 \text { (iOS) } \\
6.1 \text { (Android) } \\
2015.511 .721 .4966 \text { (Windows Phone) } \\
\end{array}$ \\
\hline \multicolumn{2}{|l|}{ Observaciones } \\
\hline Colaborador & \\
\hline
\end{tabular}

\section{Desarrollo de aplicaciones móviles}

\begin{tabular}{|l|l|}
\hline $\begin{array}{l}\text { Nombre de la } \\
\text { herramienta }\end{array}$ & Apktool \\
\hline Descripción breve & Aplicación para compilar y descompilar paquetes APK de Android \\
\hline Enlace & https://ibotpeaches.github.io/Apktool/ \\
\hline $\begin{array}{l}\text { Sistema(s) } \\
\text { operativo(s) }\end{array}$ & $\begin{array}{l}\text { Windows } \\
\text { Mac OS } \\
\text { Linux }\end{array}$ \\
\hline Precio/Licencia & Gratuita/Apache 2.0 \\
\hline Versión actual & 2.2 .1 \\
\hline Observaciones & \\
\hline Colaborador & \\
\hline
\end{tabular}




\begin{tabular}{|l|l|}
\hline $\begin{array}{l}\text { Nombre de la } \\
\text { herramienta }\end{array}$ & fastlane \\
\hline Descripción breve & $\begin{array}{l}\text { Conjunto de herramientas para automatizar la creación y el } \\
\text { lanzamiento de aplicaciones iOS y Android }\end{array}$ \\
\hline Enlace & https://fastlane.tools \\
\hline $\begin{array}{l}\text { Sistema(s) } \\
\text { operativo(s) }\end{array}$ & $\begin{array}{l}\text { iOS } \\
\text { Android }\end{array}$ \\
\hline Precio/Licencia & Gratuita/MIT \\
\hline Versión actual & 1.3 \\
\hline Observaciones & $\begin{array}{l}\text { Función de capturas de pantalla para automatizar la captura de } \\
\text { imágenes de las aplicaciones localizadas en varios dispositivos }\end{array}$ \\
\hline Colaborador & \\
\hline
\end{tabular}

\begin{tabular}{|l|l|}
\hline $\begin{array}{l}\text { Nombre de la } \\
\text { herramienta }\end{array}$ & Robotium \\
\hline Descripción breve & $\begin{array}{l}\text { Conjunto de herramientas para la automatización de tests de } \\
\text { aplicaciones nativas e híbridas de Android. Facilita la creación de } \\
\text { potentes tests automáticos de caja negra para interfaces de } \\
\text { aplicaciones de Android. }\end{array}$ \\
\hline Enlace & http://www.robotium.org \\
\hline $\begin{array}{l}\text { Sistema(s) } \\
\text { operativo(s) }\end{array}$ & Sistemas de escritorio (java) \\
\hline Precio/Licencia & Gratuita/Apache \\
\hline Versión actual & 5.6 .3 \\
\hline Observaciones & \\
\hline Colaborador & \\
\hline
\end{tabular}

\section{Otras}

\begin{tabular}{|l|l|}
\hline $\begin{array}{l}\text { Nombre de la } \\
\text { herramienta }\end{array}$ & Biscuit \\
\hline Descripción breve & $\begin{array}{l}\text { Permite crear listas de palabras seleccionadas de artículos o } \\
\text { cualquier página. Ofrece también traducciones. }\end{array}$ \\
\hline Enlace & http://getbiscuit.com/ \\
\hline $\begin{array}{l}\text { Sistema(s) } \\
\text { operativo(s) }\end{array}$ & $\begin{array}{l}\text { iOS } \\
\text { Android }\end{array}$ \\
\hline Precio/Licencia & Gratuita \\
\hline Versión actual & $\begin{array}{l}4.0 .7 \text { (iOS) } \\
4.0 .4 \text { (Android) }\end{array}$ \\
\hline Observaciones & $\begin{array}{l}\text { Rocío del Pozo Caamaño } \\
\text { rociodelpozo@gmail.com } \\
\text { Traductora e intérprete autónoma }\end{array}$ \\
\hline Colaborador & \\
\hline
\end{tabular}




\begin{tabular}{|l|l|}
\hline $\begin{array}{l}\text { Nombre de la } \\
\text { herramienta }\end{array}$ & CamScanner \\
\hline Descripción breve & $\begin{array}{l}\text { Aplicación para escanear imágenes y convertirlas directamente al } \\
\text { formato jpg }\end{array}$ \\
\hline Enlace & https://www.camscanner.com/user/download \\
\hline $\begin{array}{l}\text { Sistema(s) } \\
\text { operativo(s) }\end{array}$ & $\begin{array}{l}\text { iOS } \\
\text { Android } \\
\text { Windows Phone }\end{array}$ \\
\hline Precio/Licencia & Gratuita \\
\hline Versión actual & 3.9.16 (iOS) \\
\hline Observaciones & $\begin{array}{l}\text { Muy útil si se quiere extraer un cuadro de un libro y evitar perder el } \\
\text { tiempo escaneándolo y después recortándolo. Solo hay que sacar } \\
\text { una foto del cuadro, escoger la forma (rectangular o cuadrada) y } \\
\text { automáticamente se guarda en formato jpg para poder enviarla } \\
\text { adjunta en un correo electrónico. Va bien para la creación de } \\
\text { presentaciones, informes o para cualquier trabajo de investigación. }\end{array}$ \\
\hline Colaborador & $\begin{array}{l}\text { Olga Jeczmyk Nowak } \\
\text { olga.jeczmyk@europarl.europa.eu } \\
\text { UAB/Unidad de Terminología del Parlamento Europeo }\end{array}$ \\
\hline
\end{tabular}

\begin{tabular}{|l|l|}
\hline $\begin{array}{l}\text { Nombre de la } \\
\text { herramienta }\end{array}$ & Documents \\
\hline Descripción breve & $\begin{array}{l}\text { Aplicación de gestión de archivos e información. Además de facilitar } \\
\text { la organización de archivos, permite ver, leer y anotar casi cualquier } \\
\text { tipo de documento. También ofrece la posibilidad de subir archivos a } \\
\text { Dropbox o Google Drive para compartirlos. }\end{array}$ \\
\hline Enlace & $\begin{array}{l}\text { https://itunes.apple.com/us/app/documents-5-file-manager- } \\
\text { pdf/id364901807?mt=8 }\end{array}$ \\
\hline $\begin{array}{l}\text { Sistema(s) } \\
\text { operativo(s) }\end{array}$ & iOS \\
\hline Precio/Licencia & Gratuita \\
\hline Versión actual & 5.7 .7 \\
\hline Observaciones & \\
\hline Colaborador & $\begin{array}{l}\text { Rocío del Pozo Caamaño } \\
\text { rociodelpozo@gmail.com } \\
\text { Traductora e intérprete autónoma }\end{array}$ \\
\hline
\end{tabular}

\begin{tabular}{|l|l|}
\hline $\begin{array}{l}\text { Nombre de la } \\
\text { herramienta }\end{array}$ & F-Droid \\
\hline Descripción breve & $\begin{array}{l}\text { Repositorio de aplicaciones libres y de código abierto para la } \\
\text { plataforma Android }\end{array}$ \\
\hline Enlace & https://f-droid.org/ \\
\hline $\begin{array}{l}\text { Sistema(s) } \\
\text { operativo(s) }\end{array}$ & Android \\
\hline Precio/Licencia & Varias \\
\hline Versión actual & \\
\hline
\end{tabular}




\begin{tabular}{|l|l|}
\hline Observaciones & \\
\hline Colaborador & \\
\hline
\end{tabular}

\begin{tabular}{|l|l|}
\hline $\begin{array}{l}\text { Nombre de la } \\
\text { herramienta }\end{array}$ & Scanner Pro \\
\hline Descripción breve & $\begin{array}{l}\text { Herramienta que puede convertir cualquier foto de una página de un } \\
\text { documento a PDF }\end{array}$ \\
\hline Enlace & https://readdle.com/products/scannerpro \\
\hline $\begin{array}{l}\text { Sistema(s) } \\
\text { operativo(s) }\end{array}$ & iOS \\
\hline Precio/Licencia & $3,99 \$$ \\
\hline Versión actual & 7.1 .1 \\
\hline Observaciones & $\begin{array}{l}\text { Rocío del Pozo Caamaño } \\
\text { rociodelpozo@gmail.com } \\
\text { Traductora e intérprete autónoma }\end{array}$ \\
\hline Colaborador &
\end{tabular}

\begin{tabular}{|l|l|}
\hline $\begin{array}{l}\text { Nombre de la } \\
\text { herramienta }\end{array}$ & Stepes \\
\hline Descripción breve & Mobile Translation \\
\hline Enlace & https://www.stepes.com/ \\
\hline $\begin{array}{l}\text { Sistema(s) } \\
\text { operativo(s) }\end{array}$ & $\begin{array}{l}\text { IOS } \\
\text { Android }\end{array}$ \\
\hline Precio/Licencia & Gratuita \\
\hline Versión actual & $\begin{array}{l}4.0 \text { (Android) } \\
3.0 .4 \text { (iOS) }\end{array}$ \\
\hline Observaciones & \\
\hline Colaborador & $\begin{array}{l}\text { Miguel A. Jiménez-Crespo } \\
\text { miguelii@rci.rutgers.edu } \\
\text { Rutgers University, The State University of New Jersey }\end{array}$ \\
\hline
\end{tabular}

\begin{tabular}{|l|l|}
\hline $\begin{array}{l}\text { Nombre de la } \\
\text { herramienta }\end{array}$ & Telegram bots \\
\hline Descripción breve & Repositorio de bots de Telegram \\
\hline Enlace & https://storebot.me// \\
\hline $\begin{array}{l}\text { Sistema(s) } \\
\text { operativo(s) }\end{array}$ & Multiplataforma (Telegram) \\
\hline Precio/Licencia & Gratuita/Varias \\
\hline Versión actual & $\begin{array}{l}\text { Algunos bots de Telegram pueden ser útiles para la traducción, como } \\
\text { por ejemplo i18nbot (https://storebot.me/bot/i18nbot), para la } \\
\text { internacionalización de bots; pronunciationbot } \\
\text { (https://storebot.me/bot/pronunciationbot), para la lectura de } \\
\text { palabras; voicy (https://storebot.me/bot/voicybot), para la conversión } \\
\text { de audio a texto, o Image Translate Bot } \\
\text { https://storebot.me/bot/imagetranslatebot), para la traducción de } \\
\text { imágenes. }\end{array}$ \\
\hline
\end{tabular}




\begin{tabular}{|l|l|}
\hline Colaborador & \\
\hline
\end{tabular}

\begin{tabular}{|l|l|}
\hline $\begin{array}{l}\text { Nombre de la } \\
\text { herramienta }\end{array}$ & Zotero \\
\hline Descripción breve & Gestor de referencias bibliográficas \\
\hline Enlace & www.zotero.org \\
\hline $\begin{array}{l}\text { Sistema(s) } \\
\text { operativo(s) }\end{array}$ & Multiplataforma (web) \\
\hline Precio/Licencia & Gratuita/Libre, varias \\
\hline Versión actual & 4.0 .29 .16 \\
\hline Observaciones & $\begin{array}{l}\text { Hay varias aplicaciones en los centros de software de sistemas } \\
\text { operativos móviles que proporcionan funciones para Zotero. }\end{array}$ \\
\hline Colaborador & \\
\hline
\end{tabular}

